\title{
Ellipsometric analysis for CVD thick films on weakly absorbing substrates
}

\section{M.N. CHURAEVA}

Institute of Physical Chemistry, Academy of Sciences, Leninsky pr. 31, 117915 Moscow, Russia

\begin{abstract}
Ellipsometry is a traditional technique for the thickness and the optical constants of CVD films determination, and if in situ film control is impossible, the best way is to use spectroellipsometry. The novel method for quick estimation of the film parameters in the case of weakly absorbing thick film $(80-600 \mathrm{~nm})$ on nonabsorbing substrate, using spectroellipsometry is presented. The experimental results for electronbeam assisted CVB $\mathrm{ZrO}_{2}$ films on quartz,. PECVD diamond and $a-B / C: H$ films on $S i(100)$ are reported and the influence of substrate absorption and intermediate layers presence is discussed. Both the thickness and the wave dependence of the film optical constants were calculated from the azimuth of polarization spectra in the range of $360-660 \mathrm{~nm}$.
\end{abstract}

1. - Introduction.

Recent CVD technologies enable to produce films of various compositions, thickness and optical properties. Moreover one can alternate these characteristics just during deposition to obtain multi-layer systems. Ellipsometry is a traditional technique for such systems analysis, and if in situ surface control is impossible, for example because of high deposition rate, the best way is to study deposits using spectroeliipsometry [1].

We report here the method for thickness and optical constants determination in the case of transparent and weakly absorbing films on transparent substrate. The method is based on the azimuth of polarization measurement, so it is good not only for automatical spectroellipsometers, but for manual ones, equipped with high aperture monochromators.

2. - Theory.

The general ellipsometric equation, describing the reflection of polarized light from a film with thickness d and complex refractive index $n_{f}=n_{f}-i k_{f}$ on a transparent substrate, is [2]

$$
\operatorname{tg} \Psi \exp (i \Delta)=\left(\tilde{r}_{1}^{p}+\tilde{r}_{2}^{p} \beta\right)\left(1+\tilde{r}_{1}^{s} \tilde{r}_{2}^{s} \beta\right) /\left(\tilde{r}_{1}^{s}+\tilde{r}_{2}^{s} \beta\right)\left(1+\tilde{r}_{1}^{p} \tilde{r}_{2}^{p} \beta\right)
$$

where $\tilde{\beta}=\underset{\sim}{\exp }\left[-i 4 \pi d \lambda^{-1}\left(\tilde{n}_{\mathrm{r}}^{2}-\tilde{n}_{0}^{2} \sin ^{2} \varphi\right)^{1 / 2}\right]$

$\tilde{r}_{1}^{p}, \tilde{r}_{1}^{s}-$ Fresnel coefficients for the ambient/film interface (p,s 
denote the polarization directions parallel and perpendicular to the plane of incidence);

$r_{2}^{p}, r_{2}^{b}$ Fresnel coefficients for the film/substrate interface, $\Psi, \Delta$ - relative amplitude attenuation and relative phase shift between $p$ and $s$ components of the light wave, induced by reflection.

$n_{s}$ - refractive index of the substrate, $\varphi$ - the angle of incidence of $l i g h t$ and $\lambda$ is the wavelength.

If the film is transparent, $\Psi$ changes periodically with the wavelength within $\Psi_{-}, \Psi_{+}$limits (Fig. I)

$$
\Psi_{ \pm}=\operatorname{arctg}\left[\left(r_{1}^{p} \pm r_{2}^{p}\right)\left(1 \pm r_{1}^{s} r_{2}^{s}\right) /\left(r_{1}^{8} \pm r_{2}^{s}\right)\left(1 \pm{ }_{1}^{p} r_{2}^{p}\right)\right]
$$

which correspond to $\lambda$ values, given by

$$
\lambda_{j}=4 \pi \mathrm{d} \alpha / j, \quad j=1,2, \ldots
$$

where $\alpha=\left(n_{f}^{2}-n_{0}^{2} \sin ^{2} \varphi\right)^{3 / 2}$.

At these points $\Delta=0^{\circ}$ or $180^{\circ}$ depending on $\varphi$ value and on the optical constants of the $f i l m$ and the substrate. Positions of the neighboring extremums correlate

$$
\text { j } \lambda_{j}=(j+1) \lambda_{j+1} \text {. }
$$

and shift with the angle of incidence. By measuring $\Psi$ spectra for two different angles $\varphi_{1}$ and $\varphi_{2}$ or registering peaks positions in azimuth of polarization spectrum $\chi(\lambda)$ (for $\Psi_{ \pm}=x_{ \pm}$) one can determine the refractive index of the film from

$$
n_{f}=n_{a}\left[\left(\lambda_{\varphi 1}^{2} \sin ^{2} \varphi_{2}-\lambda_{\varphi 2}^{2} \sin ^{2} \varphi_{1}\right) /\left(\lambda_{\varphi 2}^{2}-\lambda_{\varphi 1}^{2}\right)\right]^{1 / 2},
$$

and then its thickness from eq(3). The measurements should be made in rather wide range, because it needs at least two $\Psi_{ \pm}$values to determine $j$. The accuracy of $n_{f}$ and $d$ calculations depends on the accuracy of $\lambda$ and $\varphi$ measurement and for most cases is better than $2-3 \%$. In the case of absorbing film $\beta$ is complex, the alternations of $\Psi$ become weaker and decrease with wave frequency, $\lambda_{j}$ values differ from those of transparent film. But if $n_{f} k_{r}<0.1$, the difference is negligibly small, and $k_{f}$ can be calculated from

$$
\mathrm{k}_{\mathrm{r}}=-\alpha \ln D / 4 \pi \mathrm{dn} \mathrm{r}_{\mathrm{r}}
$$

$D$ is given by the equation $a D^{2}+b D+c=0$, where $a=r_{2}^{p} r_{2}^{8}\left(r_{1}^{p} \operatorname{tg} \Psi-r_{1}^{3}\right)$,

$$
\begin{aligned}
& b=(-1)^{j}\left[\operatorname{tg} \Psi\left(r_{2}^{s}+r_{1}^{p} r_{2}^{p} r_{1}^{s}\right)-\left(r_{2}^{p}+r_{1}^{p} r_{1}^{s} r_{2}^{s}\right)\right] \\
& c=\operatorname{tg} \Psi r_{1}^{s}-r_{1}^{p} .
\end{aligned}
$$

The accuracy of $k_{r}$ determination is better than $4-5 \%$ even for $k_{f}=0.1$. 
Obviously, the approach enables direct calculation of the film characteristics, but it should be corrected if a dispersion takes place.

liet us assume that the refractive index of a film is $n_{f}$ at $\lambda_{j}$, and it is $\left(n_{f}+\Delta n_{f}\right)$ at $\lambda_{j+1}$. If $\Delta n_{f} / n_{f}<0.1$, the shift of the extremum point $\lambda_{j}$ due to dispersion will be:

$$
\Delta \lambda_{j+1}=\Delta n_{f} n_{f} \lambda_{j+1} / \alpha^{2},
$$

So, the dispersion $\delta n / \delta \lambda$ inside the interval $\lambda_{j}, \lambda_{j+1}$ is given by

$$
\delta \mathrm{n} / \delta \lambda=-\left[\alpha(j+1) / \lambda_{j}\right]^{2} \Delta \lambda_{j+1} / j n_{f},
$$

Thus, if there is $n_{f}$ dispersion, it is better to operate in the nar row range of $\lambda$. With this in mind the measurement near to the Brewster angle seems to be more preferable, for in this case we can calculate $n_{f}$ and $d$ from the equation

$$
\chi(\lambda)=0
$$

For two neighboring roots of the eq(10) it holds

$$
1 / \lambda_{i}+1 / \lambda_{i+1}=i / 2 d \alpha
$$

and the dispersion can be neglected, because the points $\lambda_{i}, \lambda_{i+1}$ are situated much closer than the points $\lambda_{j}, \lambda_{j+1}$ from eq(4). The accuracy of $n_{i}$ and $d$ determination only depends on the accuracy of $\varphi$ and $\lambda$ od justment, and for the case presented in Fig.l is: $\delta n_{f}=0.08 \%$, $\delta d=$ $0.45 \%$.

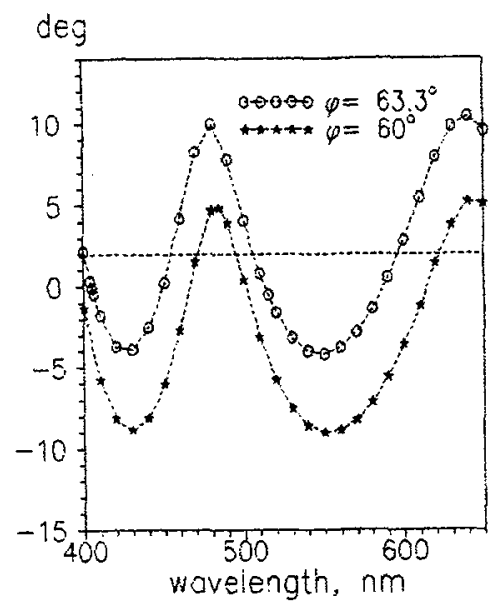

Fig.l. $x$-spectra of $\mathrm{ZrO}_{2} \mathrm{film}^{\mathrm{m}}$ on fused silica.

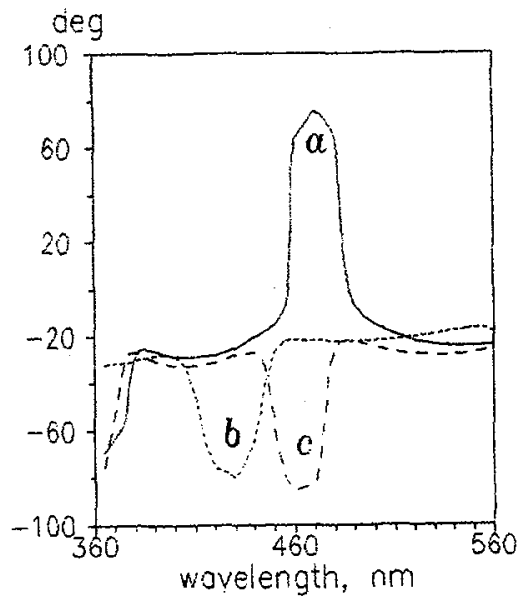

Fig. $2 x$-spectra of cVD $f i l m s$ on si, $\varphi=60^{\circ}$ : a) $n_{\hat{r}}=1.86, d=500 \mathrm{~nm}$, b) linear $n_{i}=1.46-2.0$ profile, c) $10 \mathrm{~nm}$ a-si interlayer. 
If one deals with an absorbing substrate, for example si, GaAs, other semiconductors, computer search should be performed in order to determine film parameters (especially $k_{f}$ ). We operated in accordance with the following scheme. First $\Psi, \Delta$ values were measured at two different angles of incidence and at a fixed wavelength and the solution of eqn $\left(n_{f}, k_{f}\right.$, d) was found at this wavelength by the computer searching ( $n_{s}$ values were taken from [8]). Then we varied $n_{r}$ and $k_{f}$ (d was fixed) to fit the experimental $\chi(\lambda)$ curve. $\chi$-spectrum is very sensitive to the film parameters, the presence of intermediate layers and film optical constants profile (Fig. 2) and we used it in computer simulations instead of traditional $\psi$ or $\Delta$ spectra.

\section{3. - Experimental procedure.}

The measurements have been carried out using Gaertner lll l9xuv manual ellipsoneter with Bausch \& Lomb grating monochromator ( 650 grooves $/ \mathrm{mm}$, exit slit. $1.5 \mathrm{~mm}$ ) in the range of $\lambda=360-660 \mathrm{~nm}$.

$\mathrm{ZrO}_{2}$ films were deposited on fused silica $\left(\mathrm{n}_{\mathrm{s}}=1.46\right)$ by electron-beam assisted evaporation in vacuum ( $\cong 510^{-5}$ torr) from Zr target, at the substrate temperature $100^{\circ} \mathrm{C}$, deposition rate being Inm/s. The samples were aged in air over $1.5-2$ months.

Boron-carbon and diamond like films ( $90 \% \mathrm{sp}^{3}$ bonds) were deposited on silicon wafers $S i(100)$ by $P E C V D$. Boron-carbon films were prepared in de glow discharge plasma at $0.1-5 \mathrm{~Pa}, \mathrm{~T}=50-250^{\circ} \mathrm{C}$, from carborque as

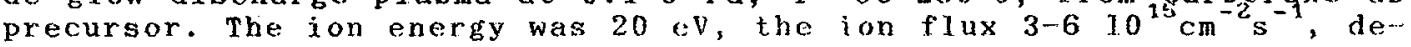
position rate about $0.4 \mathrm{~nm} / \mathrm{s}$, all the deposition procedure is described in details in $[3,4]$.
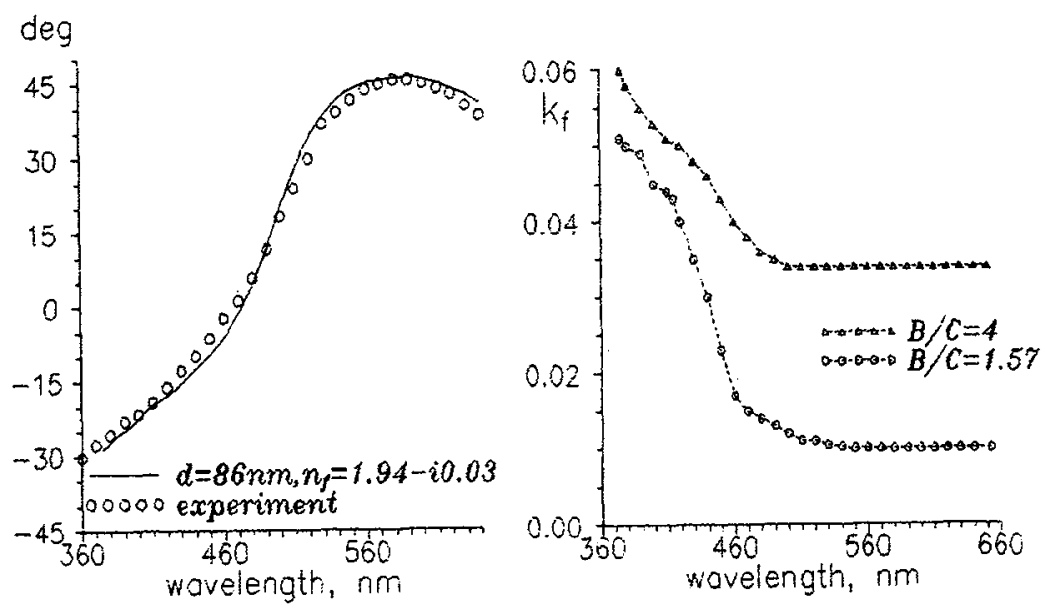

Fig. 3. X spert.ra of $a-B / C$

film on $\mathrm{S}$ i $(100), \mathrm{R} / \mathrm{C}=4, \varphi=60^{\circ}$

Eig. 4. Light absorption in PECVD a-B/C films on Si(100). All the calculations were performed on $P C 286$ AT/XT using own computer
programs, the main algorithm published in [5]. 


\section{4.- Results and discussion.}

The $\mathrm{ZrO}_{2}$ films parameters at $\lambda=590-620 \mathrm{~nm}$ were calculated using azimuth of polarization spectra at $\varphi_{1}=45^{\circ}$ and $\varphi_{2}=70^{\circ}$, they are: $n_{r}=1.96-$ i0.02, $d=513 \mathrm{~nm}$; the $\mathrm{n}_{\mathrm{f}}$ dispersion in the range $\lambda=430-477 \mathrm{~nm}$ was $\delta \mathrm{n} / \delta \lambda=510^{-4} \mathrm{~mm}$.

The $\mathrm{X}$-spectra of the $\mathrm{ZrO}_{2}$ film, measured at near to the Brewster ang-les are presented in the Fig.l, the film parameters calculated from eq(11) are: $n_{f}=1.9-i 0.01, d=571 \mathrm{~nm}$. The film porosity (1-q) was estimated from the Lorenz-Lorentz equation [6]

$$
\left(n_{f}^{2}-1\right) /\left(n_{f}^{2}+2\right)=q\left(n_{b}^{2}-1\right) /\left(n_{b}^{2}+2\right) \text {, }
$$

where $n_{b}=2.17$ is the bulk value of $\mathrm{ZrO}_{2}$ refractive index [7]. For the films investigated the porosity was $\sim 16 \%$.

optical parameters of diamond-like films were calculated using $\Psi(\lambda)$, $\Delta(\lambda)$ curves and $\chi(\lambda)$ as well, the calculations yield $n_{f}=2.255-i 0.00$

for films $33.6 \mathrm{~nm}$ thick.

$x$-spectrum of $a-B / C$ film $(B / C=4)$ on silicon is shown in Fig. 3. together with theoretical curve built using the computer calculated film parameters and silicon optical constants from [8]. The calculations showed the increasing film absorption in the short wavelength range of spectra. Although the films with high B/C ratio exhibited higher absorption (Fig. 4).

Al1 a-B/C films had refractive indices lying between those of diamond $n=2.25$ and graphite $n=1.8$. The higher $B / C$ ratio in the film was, the closer was its refractive index to that of diamond. This fact is ina good agreement with data on the films structure and the type of chemical bonds in it [9].

When studying a-B/C films produced under $500 \mathrm{~V}$ bias an intermediate a$\mathrm{Si}: \mathrm{H}$ layer was detected and its thickness was in the range from 5 to $10 \mathrm{~nm}$ for different samples.

5.- Conclusion.

The ellipsometric method for quick estimation of the film parameters in the case of weakly absorbing $80-600 \mathrm{~nm}$ films on nonabsorbing and weakly absorbing substrates is presented. It provides CVD films analysis including the determination of their thickness, optical constants and microporosity.

\section{References.}

[1] Arwin H., Aspnes D.E., Thin Solid Films 1382 (1986) 95.

[2] Azzam R.M.A.and Bashara N.M., Ellipsometry and Polarized Light, North-Holland Amsterdam (1977) 583.

[3] Kanaev A.I. et al., Dok. Acad. Nauk SSSR 318 (1991) 342

[4] Kanaev A.I., Rybakov S.Yu., Churaeva M.N, ibig.

[5] Churaeva M.N., Zorin Z.M., Interface modelling in ellipsometry. Moscow (1985) BINITI Dep.N1050-85, N1051-85.

[6] Churaeva M.N., Zorin Z.M., Persiantseva V.P., Poverkhost 2 (1985) 132 .

[7] Wood D.L., Nassan K., Appl.Opt. 2l (1982) 2978.

[8] Aspnes D.E., Studna A.A, Phys.Rev.B 2 (1983) 985.

[9] Alimov V.Kh. et al. J.Nucl.Mat. 196-198 (1992) 670 . 